\title{
EXCLUSIVE HUMAN MILK VS PRETERM FORMULA: RANDOMIZED TRIAL IN EXTREMELY PRETERM INFANTS
}

E.A. Cristofalo ${ }^{1}$, R.J. Schanler ${ }^{2}$, C.L. Blanco ${ }^{3}$, S. Sullivan ${ }^{4}$, R. Trawoeger ${ }^{5}$, U. Kiechl Kohlendorfer, ${ }^{5}$, S. Abrams $^{6}$, G. Dudel1 ${ }^{7}$, D.J. Rechtman ${ }^{8}$, M.L. Lee ${ }^{9}$, H2MF Study Group

${ }^{1}$ Pediatrics, Johns Hopkins Hospital \& School of Medicine, Baltimore, MD, ${ }^{2}$ Pediatrics, Steven and Alexandra Cohen Children's Medical Center of New York, New Hyde Park, NY, ${ }^{3}$ Pediatrics, University of Texas Health Science Center San Antonio, San Antonio, TX, ${ }^{4}$ Pediatrics, University of Florida, Gainesville, FL, USA, ${ }^{5}$ Pediatrics, Innsbruck Medical University, Innsbruck, Austria, ${ }^{6}$ Pediatrics, Baylor College of Medicine, Houston, TX, ${ }^{7}$ Pediatrics, Children's Hospital and Research Center, Oakland, ${ }^{8}$ Medical Affairs, ${ }^{9} R \& D$, Prolacta Bioscience, Monrovia, CA, USA

Background: A randomized, trial of a nutritionally adequate diet consisting solely of human milk (HM) vs preterm formula (PF) has not been conducted in extremely premature (EP) infants.

Objective: To assess the effects of HM vs PF as sole diets in EP infants.

Methods: A multicenter randomized controlled trial of EP infants (birth weight 500-1250 g) whose mothers were unable/unwilling to provide breast milk. The infants were to receive either PF or HM (pasteurized donor human milk and human milk-based human milk fortifier). The major outcomes were duration of parenteral nutrition (TPN), growth, incidence of NEC and of other morbidities.

Results: The two groups were similar in demographic and baseline characteristics. The median duration of TPN was 27 vs $36 \mathrm{~d}$ (HM, PF respectively)p $=0.04$ For surgical NEC, there were 0 vs 4 cases in the HM and $\mathrm{PF}$ groups, respectively, $\mathrm{p}=0.04$. There were no significant differences in LOS, CLD, ROP or death, however the cumulative morbidity score was lower in HM vs PF groups, $0.7 \pm 0.5 v s 1.2 \pm 1.0, \mathrm{p}=0.03$. There were no differences in weight gain or head circumference increment, but length growth velocity differed in $\mathrm{HM}(0.12 \pm 0.03)$ vs $\mathrm{PF}(0.16 \pm 0.04 \mathrm{~cm} / \mathrm{d})$ groups, $\mathrm{p}=0.006$

Conclusion: This is the first randomized trial in EP infants of exclusive HM vs PF. The significantly shorter duration of TPN and lower rate of surgical NEC support major changes in the strategy to nourish EP infants in the NICU. 